Revista Arbitrada Interdisciplinaria KOINONIA

Año IV. Vol IV. N ${ }^{\circ}$ 8. Julio - Diciembre 2019

Hecho el depósito de Ley: FA2016000010

ISSN: $2542-3088$

FUNDACIÓN KOINONIA (F.K). Santa Ana de Coro. Venezuela.

Mariannys Mariely Salón Pereira; Josía Isea

DOI: http://dx.doi.org/10.35381/r.k.v4i8.235

\title{
El emprendimiento agroalimentario y políticas públicas en Venezuela
}

\section{Agricultural entrepreneurship and public policies in Venezuela}

\author{
Mariannys Mariely Salón Pereira \\ mariannys.mmsp@gmail.com \\ Universidad Nacional Experimental Francisco de Miranda \\ Venezuela \\ https://orcid.org/0000-0003-1368-0538 \\ Josía Isea \\ josiaisea@gmail.com \\ Universidad Nacional Experimental Francisco de Miranda \\ Venezuela \\ https://orcid.org/0000-0001-8921-6446
}

Recibido: 15 de enero del 2019

Aprobado: 1 de marzo del 2019

\section{RESUMEN}

El actual artículo es una investigación que se constituye en un adelanto teórico sobre una investigación doctoral referida al emprendimiento en el sector agrícola venezolano, de ese modo, permite concebir un primer acercamiento al estado del arte, para así construir progresivamente el entramado teórico de salida, la cual debe converger hacia el aporte social - científico. Metodológicamente se abordó desde un tipo de investigación documental, entre las principales conclusiones se tiene que las políticas públicas actuales se circunscriben al fomento de la comuna como espacio productivo, es necesario realizar investigaciones en este segmento con el fin de conocer científicamente el desarrollo, alcance, beneficios, debilidades, para así, potenciar o tomar correctivos que permitan desde la comuna, afianzar el emprendimiento como un estilo de vida que contribuya a transcender el modelo económico del rentismo petrolero en una economía productiva desde lo endógeno.

Descriptores: Economía agraria; Economía rural; Desarrollo agrícola; Política agraria. Política ambiental. 


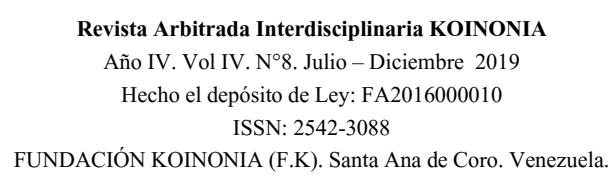

Mariannys Mariely Salón Pereira; Josía Isea

\section{SUMMARY}

The current article is an investigation that constitutes a theoretical advance on a doctoral research related to entrepreneurship in the Venezuelan agricultural sector, in this way, allows to conceive a first approach to the state of the art, which will gradually build the theoretical framework of output, which must converge towards the social - scientific contribution. Methodologically it was approached from a type of documentary research, among the main conclusions is that current public policies are limited to the promotion of the commune as productive space, it is necessary to conduct research in this segment in order to scientifically know the development, scope, benefits, weaknesses, in order to strengthen or take corrective measures that allow the commune to consolidate entrepreneurship as a lifestyle that contributes to transcending the economic model of oil rent in a productive economy from the endogenous.

Descriptors: Agrarian economy; Rural economy; Agricultural development; Agrarian policy. Environmental policy.

\section{INTRODUCCIÓN}

El emprendimiento en las comunidades agrícolas venezolanas, se torna importante por cuanto representa un historial ancestral de conocimientos que son meritorios de ser estudiados y estructurados en conocimiento científico. Desde la era poscolonial venezolana, ha existido por parte de las personas que habitan el medio rural, el interés por desarrollar las fuerzas productivas del campo, bien sea de orden agrícola o ganado, esto en gran parte ha sido generado por el interés de los productores, los cuales aplicando el conocimiento ancestral han podido cultivar tierras y criar animales, con el paso del tiempo, la modernización ha permitido incorporar la tecnificación y medicina veterinaria como valores agregados a la producción.

Esto ha permitido, la generación de emprendedores en el sector agrícola - pecuario venezolano, los cuales paradójicamente no han sido proyectados en importancia por los sectores de poder la sociedad, es decir, se ha menospreciado los emprendimientos rurales por darle mayor interés a los del sector urbano. Es de destacar que el actual papel de trabajo, procura la generación de un análisis sobre el tema del 


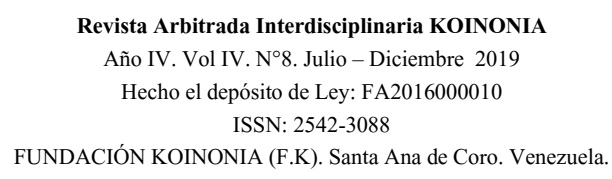

Mariannys Mariely Salón Pereira; Josía Isea

emprendimiento agroalimentario, por cuanto esto permitirá develar prejuicios existentes en el área.

Propiciando así, un acercamiento a la construcción de nuevas categorías de estudio que podrían conducir hacia la estructuración de teorías construidas desde la perspectiva de los emprendedores en el área agrícola, para esto es importante destacar la definición de emprendimiento empleada por (Marulanda, Correa y Mejía, 2009) quienes señalan que "el emprendimiento es una de las características que determina el crecimiento, la transformación y el desarrollo de nuevos sectores económicos de una región o un país, siendo el ser humano el principal pilar", (p. 153).

Desde la perspectiva planteada, construir la teoría desde la imagen de emprendimiento que poseen los productores agrícolas que ha podido desarrollar una idea de negocio y crecer en el tiempo, permitirá contextualizar aportes teóricos al entramado bibliográfico venezolano, con énfasis en el sector político, debido que las leyes y políticas son generadas en ocasiones por personas que desconocen el tema del emprendimiento, mucho más si es del sector rural.

En este sentido, se estudia el emprendimiento rural agrícola, así como también las políticas públicas existentes para el desarrollo y progreso de tal sector, con lo cual, podría verse configurada posibles brechas que imposibilitan que existan acciones consensuadas al pleno fomento de la actividad económica rural agrícola con perspectiva en el emprendimiento, el cual debe nacer desde las ideas de negocio de los habitantes del área agrícola pecuario. La visión de brechas entre el emprendimiento rural y urbano, podría tener su fundamento en la medida como se ha estudiado la economía, en este sentido, (Heynig, 1982) plantea que

El enfoque modernizante formulado principalmente desde la economía neoclásica también tienen como concepción básica la dicotomía tradicional moderno. Para este enfoque los países subdesarrollados tienen dos sectores separados y diferentes: "el sector moderno capitalista industrial, receptivo al cambio, orientado al cambio y cuyo comportamiento persigue maximizar sus ganancias y el sector tradicional agrícola y estancado, basado en la producción de subsistencia, con escasos excedentes para la 


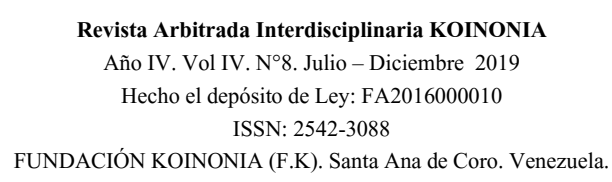

Mariannys Mariely Salón Pereira; Josía Isea

comercialización, con una significativa preferencia por una vida ociosa y escaso interés por obtener ganancias." (p. 120).

En el imaginario social venezolano, podría aun permanecer la cosmovisión descrita por Heynig, lo cual generaría la permanencia de la discriminación hacia el emprendimiento en el sector rural agrícola, lo cual además se contradice con la realidad en donde el sector agrícola cuando es apoyado con políticas públicas acertadas, tiende a ser productivo, rentable, es por lo concebido que se genera por parte de la investigadora, la interrogante sobre ¿el rol del emprendimiento agroalimentario y las políticas públicas en Venezuela?

Desde tal premisa, se explora como el emprendimiento rural agrícola contribuye al progreso nacional y como este a su vez, es apoyado por una políticas públicas que permitan sostener en el tiempo las ideas de negocio propiciadas por las personas que hacen vida en el campo venezolano, por consiguiente, permitirá concebir un acercamiento heurístico - hermenéutico sobre el fenómeno planteado, abriendo la posibilidad de conocer desde la realidad social de quienes protagonizan día a día la economía agropecuaria.

La investigación se constituye en un adelanto teórico sobre una investigación doctoral referida al emprendimiento agrícola venezolano, de ese modo, permite concebir un primer acercamiento al estado del arte, lo cual permitirá construir progresivamente el entramado teórico de salida, la cual debe converger hacia el aporte social - científico mediante la teorización del fenómeno planteado, esto además, germinará en conocimientos que podrán ser compartidos, discutidos, desde los actores sociales, protagonistas del campo venezolano, quienes han generado emprendimiento a pesar de las múltiples adversidades que padecen.

\section{Objetivo de la investigación}

Analizar el rol del emprendimiento agroalimentario y las políticas públicas en Venezuela. 


\section{DESARROLLO}

\section{Antecedentes}

En este sentido, (Giacalone, Hernández y Zerpa, 2010), realizaron un artículo de investigación, donde plantean

Que existen factores importantes que conspiran para entorpecer el desempeño del SAV y el logro de los objetivos que se propone el Estado venezolano mediante su política de financiamiento. Entre esos factores destacan la desconfianza de los actores privados con respecto a los objetivos del Estado para la agricultura, así como la contradicción entre las políticas implementadas por éste. (p. 167).

Se evidencia la existencia de una política de financiación por parte del Estado Venezolano al sector agroalimentario ha existido en las últimas décadas, sin embargo, el mismo ha sido enfocado hacia un segmento productivo como lo es el privado, denotándose que este puede estar conformado por las grandes empresas y no necesariamente por los pequeños productores o emprendedores, quienes parten de una idea de negocio que en ocasiones es motorizada por la fe y esperanza de realizar un buen negocio productivo, lo que deja abierta la brecha de posibilidades para los emprendimientos, por cuanto, no ser percibe formación gerencial ni técnica en el estudio referencial como parte de la política pública.

La existencia de brechas entre las políticas empleadas y el emprendimiento agroalimentario, deja vacíos teóricos que podrían ser develados en la investigación doctoral a realizar, por cuanto al trabajar con los emprendedores, éstos podrían brindar la información pertinente para categorizar aristas que permitan coadyuvar a la realización de teorías que permeen hacia la futura construcción de políticas públicas con pertinencia a la potenciación de los emprendedores noveles del sector agroalimentario.

En complemento de lo planteado, (Peña Aldazoro, 2018) generó un artículo de investigación, donde plantea que, 


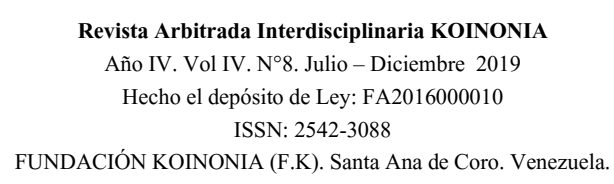

Mariannys Mariely Salón Pereira; Josía Isea

Las políticas alimentarias en Venezuela han tenido un efecto positivo en la población; emprendiendo una interesante política de inclusión, reconocida por organizaciones internacionales bajando índices de pobreza, la inclusión social es un hecho y ha tenido impacto importante en el nuevo ordenamiento de estratos sociales venezolano. (p. 60).

Se destaca la apertura de las políticas públicas hacia la financiación de los productos finales consumidos por la población, sin embargo, no se genera la reflexión sí estos han sido producidos por emprendedores venezolanos o por grandes empresas nacionales 0 internacionales, de ese modo, es pertinente enfocar el actual estudio, a descubrir evidencias que permitan confrontar cual ha sido la magnitud de apoyo a los emprendedores noveles del sector agroalimentario en materia de políticas públicas, lo cual brinda la oportunidad para realizar futuros aportes teóricos en el marco de la teorización de la tesis doctoral referida al emprendimiento en el sector agrícola venezolano.

\section{Referencial teórico}

El emprendimiento epistémicamente puede ser concebido desde diversas ópticas, sin embargo, para el presente estudio, se enfoca hacia el segmento agroalimentario, específicamente a emprendedores noveles, quienes han aportado al crecimiento económica, desde su propia inventiva o tenacidad para desarrollar negocios sin que en ocasiones posean la formación especializada para tal fin, por consiguiente, se iniciará desde la perspectiva de la Comisión Nacional de la Micro y Pequeña Empresa (CONAMYPE), quien plantea que el

Emprendimiento es una manera de pensar y actuar orientada hacia la creación de riqueza para aprovechar las oportunidades presentes en el entorno o para satisfacer las necesidades de ingresos personales generando valor a la economía y a la sociedad. Mientras que el Emprendedor: Es una persona con capacidad de innovar; entendida esta como la capacidad de generar bienes y servicios de una forma creativa, metódica, ética, responsable y efectiva. (CONAMYPE, 2014, p. 13). 
En complemento de lo planteado, el emprendimiento debe ser desarrollado por el emprendedor en un marco de ciudadanía global, donde juegue a la formula ganar ganar, es decir, realiza la actividad económica mediante un clima de respeto hacia los consumidores y medio ambiente, esto propicia la posibilidad de generar emprendimientos agroalimentarios sustentables y sostenibles, en donde se promuevan practicas económicas afines al progreso integral de la sociedad, constituyéndose en una real oportunidad de crecimiento en favor de las economías emergentes. Así mismo, la (CONAMYPE, 2014, p. 13), plantea que el emprendimiento se clasifica en:

1. Emprendimiento por Necesidad: Acción empresarial iniciada por personas que al momento de tomar la decisión de poner en marcha una actividad económica lo hicieron motivados por la falta de ingresos necesarios para su subsistencia (o por el deseo de obtener una fuente de ingreso adicional). Esta categoría se divide en dos tipos: emprendimiento de subsistencia y emprendimiento tradicional, las cuales se presentan a continuación:

1.1. Emprendimiento de Subsistencia: Acción empresarial dirigidas a generar ingresos diarios para vivir (autoempleo), sin una planificación o sin una visión de crecimiento y que tiende a no generar excedentes.

1.2. Emprendimiento Tradicional: Acción empresarial dirigida a la generación de ingresos que cuentan con una estructura organizacional y que utilizan el conocimiento técnico para la generación de excedentes que permiten la acumulación. Tienden a desarrollar su actividad en la formalidad, en mercados y sectores tradicionales de la economía sin elementos diferenciadores en sus productos y servicios.

2. Emprendimiento por Oportunidad: Acción empresarial iniciada por personas que al momento de tomar la decisión de poner en marcha una actividad económica lo hicieron motivados por la identificación de una oportunidad de mercado. Esta categoría se divide en dos tipos: 


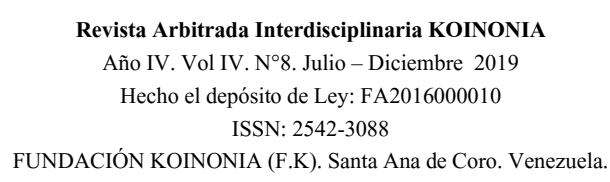

Mariannys Mariely Salón Pereira; Josía Isea

2.1. Emprendimiento Dinámico: Acción empresarial con alto potencial de crecimiento donde el uso del conocimiento, la gestión tecnológica y del talento humano, el potencial de acceso a recursos de financiación/inversión y una estructura de gobierno corporativo les permite generar una ventaja competitiva y diferenciación en sus productos o servicios.

2.2. Emprendimiento de Alto Impacto: Empresas con capacidad para transformar y dinamizar las economías a través de procesos sistemáticos de innovación y generación de empleo. Es una empresa que crece rápida y sostenidamente, ya que cuenta con altos niveles de financiación o de inversionistas.

El emprendimiento agroalimentario podría ser desarrollado desde ambos escenarios de la clasificación descrita, sin embargo, la experiencia a priori de la investigadora, indica que la gran mayoría de los emprendimientos ocurre en el primer segmento, los cuales tienden progresar por la tenacidad de las personas que lo desarrollan, siendo este un criterio a ser investigado durante el trabajo de investigación doctoral, es decir, como nacen, se mantienen en el tiempo, las empresas que han nacido de emprendimiento por necesidad, mientras que en el actual papel de trabajo se focaliza en estudiar el apoyo desde las políticas públicas venezolanas, lo cual, desde esa arista no se genera distinción de estudio hasta el momento.

\section{Enfoques actuales de emprendimiento}

En este sentido, la tendencia actual gira hacia visiones de articular la posibilidad de generar emprendimientos, la primera es conocida como: Ecosistema de emprendimiento,

Es la comunidad de negocios, apoyada por un con texto público de leyes y prácticas de negocios formada por una base de organizaciones y personas interactuantes que producen y asocian ideas de negocios, habilidades, recursos financieros y no financieros que resultan en empresas dinámicas (CONAMYPE, 2014, p. 15). 
Este tipo de emprendimiento tiene apoyo desde instituciones que funcionan como incubadoras o creadoras de emprendimientos, en este sentido, (Nuñez Torrón, 2017) complementa diciendo que "por ecosistema emprendedor, se entiende todo aquel contexto y entorno que facilitan el surgimiento de empresas y proyectos empresariales" (p. 1). Esto implica la existencia de políticas públicas destinadas a la confección de nuevas empresas mediante el apoyo integral a los emprendedores, siendo esto propicio para el sector novel agroalimentario, el cual debe ser apoyado no solo con financiamiento, sino, con formación especializada a fin de generar la sustentabilidad y sostenibilidad pertinente en pro de asegurarse en el tiempo, una empresa productiva y rentable. 
Liderazgo

* Apoyo inequívoco

* Legitimidad social

* Puerta abierta a las propuestas

* Estrategia emprendedora

* Urgencia, crisis y retos

\section{Primeros clientes}

* Innovadores tempranos para pruebas de concepto

* Experiencia en productividad

* Clientes referencia

* Primeros comentarios

* Canales de distribución

\section{Redes}

"Redes de empresarios

* Redes de diáspora

* Corporaciones

multinacionales

Trabajo

* Capacitado y no capacitado

"Emprendedores seriales

* Familia de última generación

Instituciones educativas

* Grados generales (profesionales y académicos)

- Capacitación especifica de emprendimiento

\section{Infraestructura}

"Telecomunicaciones

* Transporte y logística

* Energía

*Zonas, centros de incubación, cluster's

\section{Profesiones de apoyo}

* Legal

* Contabilidad

* Banca de Inversión

${ }^{*}$ Asesores técnicos expertos

\section{Gobierno}

* Instituciones, Ejem.: Inversión, apoyo

" Apoyo financiero, Ejem.: I+D, fondos jump start

* Marco regulatorio de incentivos, Ejem.: Beneficios fiscales

* Institutos de investigación

* Legislación amigable de riesgo, Ejem.: Bancarrota, imposición de contratos, derechos de propiedad y trabajo

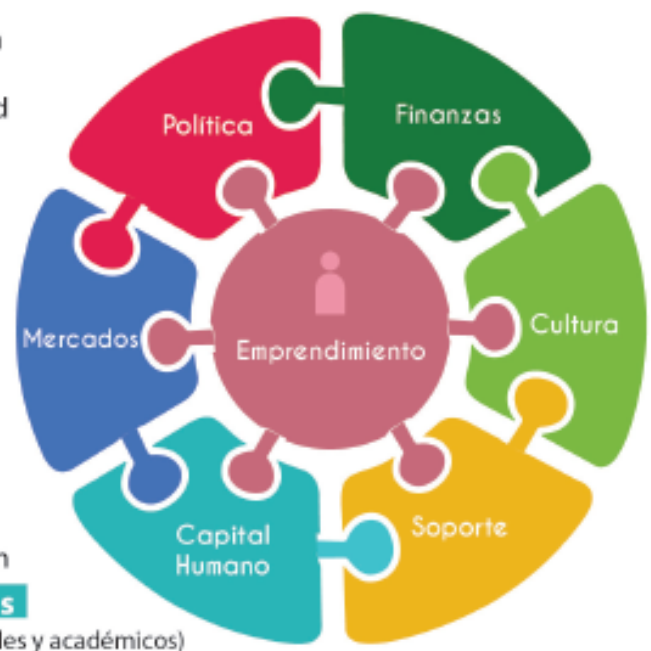

Capital financiero

* Micro préstamos

* Ángeles inversionistas, amigos y familia

* Capital de riesgo en etapa cero

* Fondos de capital de riesgo

* Capital privado

* Marcados públicos de capital

* Deuda

\section{Historias de éxito}

* Exitos visibles

* Generación de riqueza para

los fundadores

${ }^{*}$ Reputación Internacional

\section{Normas sociales}

* Tolerancia al riesgo, errores y

fracaso

* Innovación, creatividad y experimentación

${ }^{*}$ Creación de riqueza

* Ambición, esfuerzo y deseo

\section{Instituciones no gubernamentales}

* Promoción del emprendimiento en organizaciones

sin ánimos de lucro

* Conferencias

* Competencias de plan de negocios

* Asociaciones amigables a emprendedores

Figura 1. Características del ecosistema emprendedor. Planteado por: Babson Global's Entrepreneurship Ecosystem Project, Dr. Daniel Isenberg. Tomado de (CONAMYPE, 2014, p. 18).

Las características presentadas en la figura, evidencian que el ecosistema de emprendimiento, es un sistema complejo, el cual requiere de apoyo y seguimiento para poder establecer una idea de negocio que sea provechosa para las partes interesadas. Uno de estos apoyos es el gubernamental, siendo indispensable para tal fin, la 


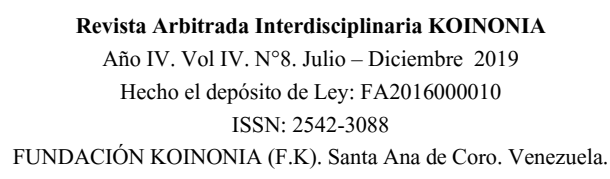

Mariannys Mariely Salón Pereira; Josía Isea

existencia de políticas públicas que permeen hacia el éxito y consolidación del emprendimiento.

Otro enfoque del emprendimiento, es conocido como "Fomento de la cadena de valor del emprendimiento", en síntesis procura apoyar el emprendimiento mediante la elaboración de un valor agregado a la producción, la cual permite concebir diferenciación en el mercado. Este enfoque resulta del intercambio de apoyo de instituciones públicas y privadas que permiten establecer alianzas para el desarrollo de un determinado sector productivo. En este sentido, se puede citar la experiencia desarrollada en Nicaragua donde Alemania contribuyó en el fomento del valor del cacao, tal como exponen (Navarro Prado, Aguilar, López Sampson, Breuer, 2013)

La Cooperación Alemana inició acciones de fomento de la cadena de valor de cacao en Nicaragua desde el año 1990 a través de la organización no gubernamental Pro Mundo Humano en el marco de una colaboración con la empresa privada alemana Ritter SPORT, apoyando a grupos de productores de cacao que posteriormente conformaron la Cooperativa Cacaonica en Waslala, Matagalpa. Las principales acciones apoyadas fueron el fomento y mejoramiento de la producción de 350 ha de cacao bajo sistema agroforestal, certificación y comercialización de cacao. (p. 12).

Este enfoque requiere del diálogo entre las instituciones públicas y privadas para construir un plan de trabajo que permita mediante políticas públicas establecidas, desarrollar el emprendimiento más allá de la mera producción de un determinado rubro, producto o servicio, por cuanto se busca mejorar el rendimiento de este, hasta incluso lograr estándares de certificación de calidad, lo cual permite generar mayor atractivo al emprendimiento, debido que se ven beneficiadas las partes involucradas, pero sobre todo los emprendedores quienes reciben formación especializada para alcanzar el nivel de calidad requerido.

El razonamiento efectual o enfoque efectual, es un enfoque del emprendimiento el cual se basa en el ensayo y el error, pero evaluando sobre la marcha para conocer los errores, realizar los ajustes necesarios, hasta que el mismo logre la "perfección". Este tipo de emprendimiento es usado cuando no existe referencia en el mercado, es decir, 


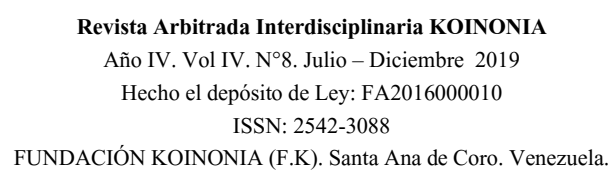

Mariannys Mariely Salón Pereira; Josía Isea

cuando ha sido poco o nula la exploración que se ha realizado, de ese modo, la (CONAMYPE, 2014), plantea que

El enfoque efectual es adecuado para aquellos emprendimientos que representan una alta incertidumbre, porque a mayor conocimiento se tenga de las variables del negocio a partir de la experimentación, se podrá descubrir más fácilmente las claves que darán forma al proyecto, a través de la acción propia.

Este tipo de emprendimiento es usado por los emprendedores noveles cuando deciden iniciar una idea de negocio sin tener estudios de mercado que le permitan conocer previamente el comportamiento del potencial cliente frente al producto o servicio que pretende producir, es quizás el emprendimiento realizado basado en la esperanza de sacar adelante un negocio.

Esto podría representar fracasos en el emprendimiento sí al errar, no se evalúa para aprender, así como también cuando no se cuenta con el capital necesario para reinvertir y relanzar el negocio. Sin embargo, este tipo de emprendimiento realizado en base a políticas públicas que permitan mediante la investigación empresarial, apoyar las ideas de emprendimiento que se enrumban por este enfoque, por cuanto esto permitirá caracterizar el mercado a explorar, así como aprender de las múltiples variables que existen en el mismo, constituyéndose así, una posibilidad de crecimiento sí se observa desde una perspectiva de aprendizaje continuo.

El enfoque DesignThinking (DT) o pensamiento de diseño, es uno de los emprendimientos que requieren mayor innovación y trabajo en equipo para contribuir en la transcendencia de la idea de negocios. En este sentido, (Brown, 2017), explica que

El pensamiento de diseño es un enfoque de la innovación centrado en el ser humano que se basa en el conjunto de herramientas del diseñador para integrar las necesidades de las personas, las posibilidades de la tecnología y los requisitos para el éxito empresarial. (p. 1).

Se enfoca en la empatía, lluvia de ideas, para generar desde el trabajo en equipo, las diversas posibilidades a la resolución de problemas mediante la innovación, siendo esta, su principal aporte al emprendimiento. Podría señalarse que se basa en un 
enfoque desde el pensamiento innovador para conocer los multifactores que integran el mercado y así, generar estrategias que propicien el empoderamiento del mercado, de acuerdo a la idea de negocio generada.

Este enfoque podría ser desarrollado de modo exclusivo por los emprendedores o ser complementario con otro enfoque al ser usado como estrategia que permita evaluar e innovar para la resolución de temas concretos en la empresa y mercado. Este tipo de enfoque requiere en los emprendedores noveles, el apoyo en la formación para poder realizarlo efectivamente y no que el mismo se pueda constituir en improvisaciones para la organización, viéndose afectada la calidad y productividad.

Enfoque de modelo de negocios, es otra perspectiva para desarrollar emprendimientos, desde la cual, (Osterwalder y Pigneur, 2017) señalan que

Entendemos que un modelos de negocios puede ser descrito a través de nueve bloques que muestran la lógica de cómo una empresa pretende hacer dinero. Los nueve bloques cubren las cuatro áreas principales de un negocio: clientes, oferta, infraestructura y viabilidad financiera. El modelo de negocios es como el plano para una estrategia a implementar a través de las estructuras de la organización, sus procesos y sistemas.

Se expresa la ruta mediante el cual, el emprendedor pretender generar la idea de negocios con la finalidad que esta pueda ser desarrollada productivamente según el mercado abordado, es allí donde cada uno de los nueve bloques juegan un papel importante para desarrollar el negocio, a su vez que interactúan dinámicamente entre sí para confluir en el cumplimiento de las metas propuestas, de ese modo, se presentan los nueve bloques en forma de infografía: 


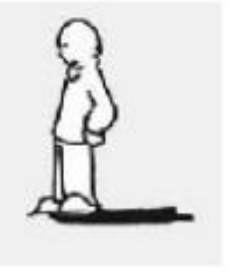

1 Segmentos de mercado

Una empresa atiende a uno o varios segmentos de mercado.

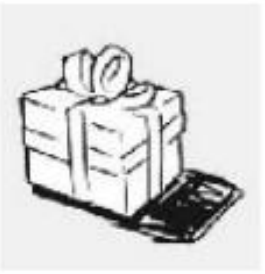

2 Propuestas de valor

Su objetivo es solucionar los problemas de los clientes y satisfacer sus necesidades mediante propuestas de valor.

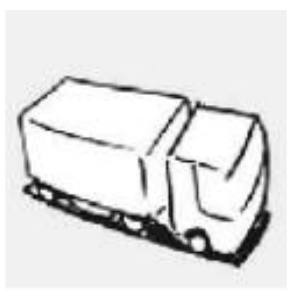

3 Canales

Las propuestas de valor llegan a los clientes a través de canales de comunicación, distribución y venta.

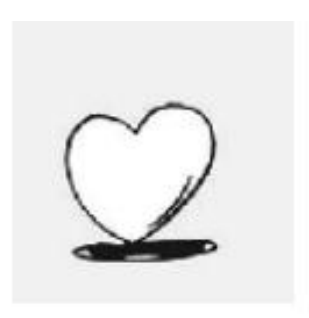

4 Relaciones con clientes

Las relaciones con los clientes se establecen y mantienen de forma independiente en los diferentes segmentos de mercado.

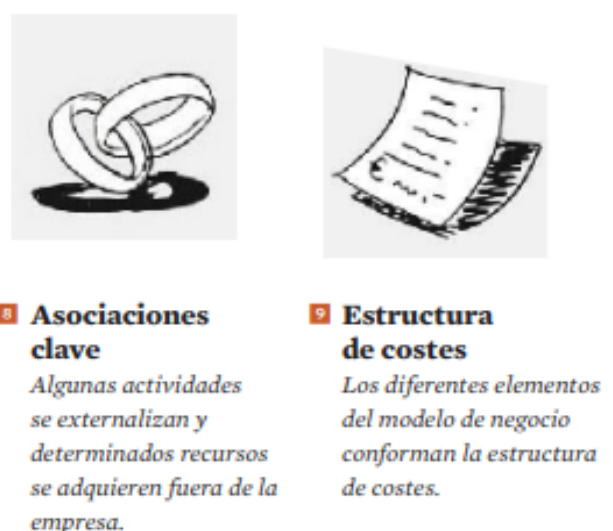

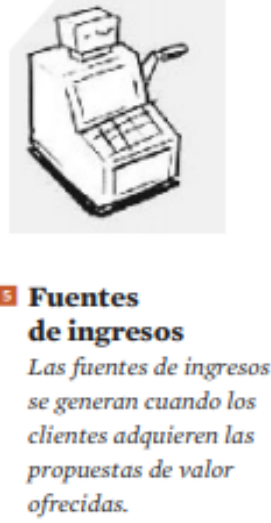

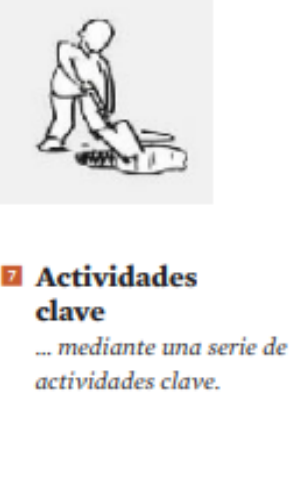

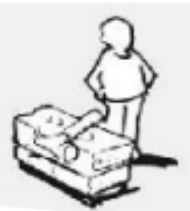

Recursos clave

Los recursos clave son los activos necesarios para ofrecer y proporciona descritos.

Figura 2. Infografía los nueve módulos. Tomado de (Osterwalder y Pigneur, 2017, p. 3).

\section{Enfoque de formación de competencias de emprendimiento desde la educación universitaria.}

Este enfoque tiene su experiencia en un proceso investigativo piloto de la Universidad La Salle en Colombia, mediante un proceso pedagógico de reflexión - acción, se articuló la configuración en los estudiantes en aprender técnicas empresariales, así como investigación, sumado al conocimiento empírico de ser agricultores. Para tal fin la universidad realizó una convocatoria abierta para todos los jóvenes que desearan 
formarse en el plan piloto, el cual fue desarrollado en los últimos cuatrimestres de la carrera de ingeniería agronómica, ante esto, (Taborda y Sosa, 2014), plantean que,

La propuesta de la Universidad de La Salle para el programa de ingeniería agronómica ha desarrollado un currículum integral que se enriquece mediante las prácticas productivas en el campus de la extensión Yopal y la práctica en zona de origen, ofreciendo a sus estudiantes diferentes mecanismos para aplicar las herramientas adquiridas en los espacios académicos en el diseño y materialización de un agronegocio con visión de generar desarrollo local. (p. 61).

La vertiente central del proyecto, considera que los emprendedores no nacen, se hacen, de ese modo, cobra valor la formación de los jóvenes en emprendedores en el agronegocio, por consiguiente realizaron una adecuación curricular que permitiera incorporar a los jóvenes campesinos a la formación universitaria, esto permitió combinar formación técnica teórica y práctica, desde la creación de la idea de negocio hasta su desarrollo, para lo cual, realizaron el siguiente diagrama de flujo: 


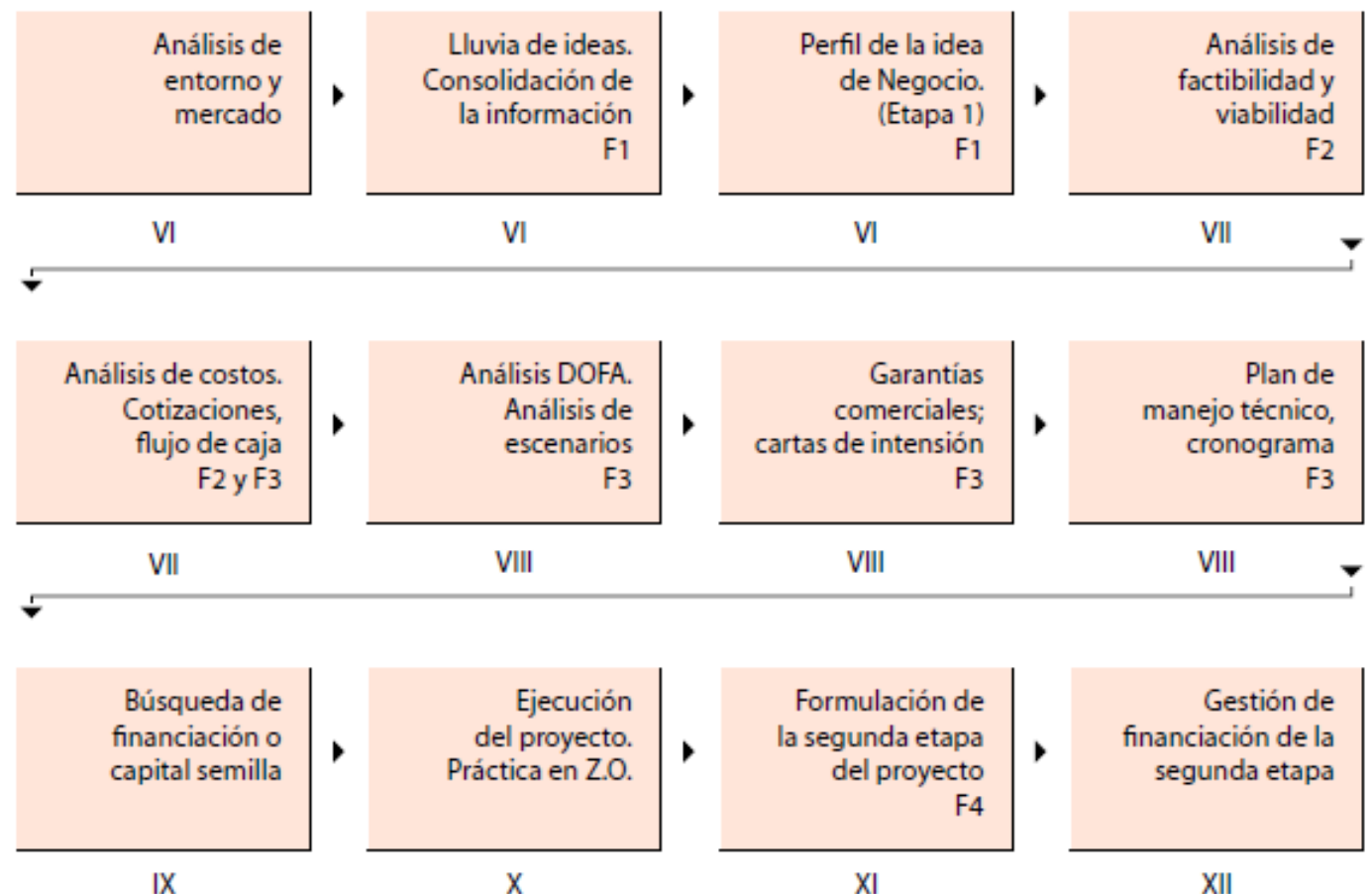

Figura 3. Diagrama de flujo del proceso de formulación del proyecto productivo. Tomado de (Taborda y Sosa, 2014, p. 56).

El diagrama de flujo, muestra como a partir del VI (sexto) cuatrimestre de la carrera de ingeniería agronómica, se realizó una adaptación curricular que permitió generar un emprendimiento mediante la realización de un proyecto productivo, en la medida que avanzaban los estudiantes adquirían competencias para articular exitosamente el emprendimiento, lo que se destaca de esta iniciativa es el apoyo de una institución de educación superior, la cual contribuyó en cambiar paradigmas formativos hacia uno que integrará la visión de emprendimiento, lo cual podría constituirse en una política pública replicable por el resto de las universidades. 


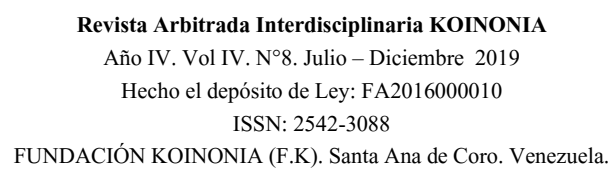

Mariannys Mariely Salón Pereira; Josía Isea

\section{Políticas públicas en el sector agroalimentario venezolano.}

Con la finalidad de sintetizar el desarrollo de políticas públicas enfocadas al sector agroalimentario, se abordará el período desde 1999 cuando entra un nuevo marco constitucional en el país hasta la fecha. De ese modo, se podrá concretar sobre los aportes y avances hacia el sector agroalimentario en los últimos años, concatenándose además con la aparición de nuevos enfoques de emprendimiento a nivel mundial, fenómeno que permitirá generar análisis sobre la situación actual de políticas que fomenten el emprendimiento en el sector agroalimentario, sobre todo en los sectores rurales poco explorados por el mercado, así como en emprendedores noveles. Es así que la (Constitución de la República Bolivariana de Venezuela, 1999) determina en su

Artículo 112. Todas las personas pueden dedicarse libremente a la actividad económica de su preferencia, sin más limitaciones que las previstas en esta Constitución y las que establezcan las leyes, por razones de desarrollo humano, seguridad, sanidad, protección del ambiente $u$ otras de interés social. El Estado promoverá la iniciativa privada, garantizando la creación y justa distribución de la riqueza, así como la producción de bienes y servicios que satisfagan las necesidades de la población, la libertad de trabajo, empresa, comercio, industria, sin perjuicio de su facultad para dictar medidas para planificar, racionalizar y regular la economía e impulsar el desarrollo integral del país (p. 29).

Lo acordado por el artículo permite la generación de iniciativas de emprendimiento, siempre y cuando estas se ajusten a lo establecido en el marco legal vigente, esto permite que cualquier emprendedor pueda generar una idea de negocio y desarrollarla según el enfoque empresarial de su preferencia, por consiguiente la iniciativa privada es fomentada desde lo expuesto. Así mismo, la (Constitución de la República Bolivariana de Venezuela, 1999) establece en su

Artículo 118. Se reconoce el derecho de los trabajadores y trabajadoras, así como de la comunidad para desarrollar asociaciones de carácter social y participativo, como las cooperativas, cajas de ahorro, mutuales y otras formas asociativas. Estas asociaciones podrán desarrollar cualquier tipo de actividad económica, de conformidad con la ley. La ley reconocerá las especificidades de estas organizaciones, en especial, las relativas al acto cooperativo, al trabajo asociado y su carácter generador de beneficios 


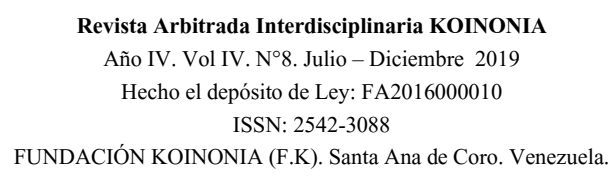

Mariannys Mariely Salón Pereira; Josía Isea

colectivos. El Estado promoverá y protegerá estas asociaciones destinadas a mejorar la economía popular y alternativa (p. 30).

El Estado privilegia el enfoque de cooperativas y el trabajo asociado como una manera alterna de mejorar la economía, esto implica generar sinergia entre un grupo de personas con el fin de llevar a cabo un emprendimiento en conjunto, siendo otro modelo empresarial que se puede generar desde una perspectiva del emprendimiento agroalimentario.

El marco constitucional descrito, permite la generación de emprendimientos desde lo individual y colectivo, opciones que permiten conciliar la posibilidad de articular políticas públicas en favor de generar riquezas económicas desde perspectivas enfocadas al desarrollo integral del país. Complementando lo planteado, la (Constitución de la República Bolivariana de Venezuela, 1999) plantea en su "Artículo 129. Todas las actividades susceptibles de generar daños a los ecosistemas deben ser previamente acompañadas de estudios de impacto ambiental y sociocultural" (p. 33).

Por consiguiente, las ideas de emprendimiento agroalimentario a generarse, deben tener en consideración el impacto ambiental y cultural que puedan generar, para esto es necesario contar con los estudios requeridos, lo cual podrían ser complementado con el estudio de mercado, así los emprendedores tendrán la oportunidad de conocer en amplitud el espacio societal donde desarrollarán su emprendimiento. Así mismo, la (Constitución de la República Bolivariana de Venezuela, 1999) establece el sistema socioeconómico, en los siguientes artículos relacionados con el emprendimiento:

Artículo 299. El régimen socioeconómico de la República Bolivariana de Venezuela se fundamenta en los principios de justicia social, democracia, eficiencia, libre competencia, protección del ambiente, productividad y solidaridad, a los fines de asegurar el desarrollo humano integral y una existencia digna y provechosa para la colectividad. El Estado, conjuntamente con la iniciativa privada, promoverá el desarrollo armónico de la economía nacional con el fin de generar fuentes de trabajo, alto valor agregado nacional, elevar el nivel de vida de la población y fortalecer la soberanía económica del país, garantizando la seguridad jurídica, solidez, dinamismo, sustentabilidad, permanencia y equidad del crecimiento de la economía, 


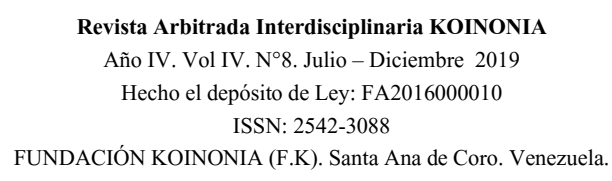

Mariannys Mariely Salón Pereira; Josía Isea

para lograr una justa distribución de la riqueza mediante una planificación estratégica democrática, participativa y de consulta abierta (p. 79).

Lo expuesto permite la generación de políticas públicas en el orden mixto (público y privado) con el fin de generar condiciones para el normal funcionamiento de empresas nacidas del emprendimiento, así mismo, en el

Artículo 305. El Estado promoverá la agricultura sustentable como base estratégica del desarrollo rural integral a fin de garantizar la seguridad alimentaria de la población; entendida como la disponibilidad suficiente y estable de alimentos en el ámbito nacional y el acceso oportuno y permanente a éstos por parte del público consumidor. La seguridad alimentaria se alcanzará desarrollando y privilegiando la producción agropecuaria interna, entendiéndose como tal la proveniente de las actividades agrícola, pecuaria, pesquera y acuícola..., (p. 80).

Se propicia la diversidad de oportunidades para generar ideas de negocios en lo relacionado al sector agroalimentario, en este sentido, al ser la alimentación un tema de seguridad nacional, se deben fomentar políticas publicas asertivas y efectivas que permeen en la consolidación de los diversos emprendimientos realizados con el fin de proporcionar alimentos y oportunidades de crecimiento económico. Por otro lado, el

Artículo 306. El Estado promoverá las condiciones para el desarrollo rural integral, con el propósito de generar empleo y garantizar a la población campesina un nivel adecuado de bienestar, así como su incorporación al desarrollo nacional. Igualmente fomentará la actividad agrícola y el uso óptimo de la tierra mediante la dotación de las obras de infraestructura, insumos, créditos, servicios de capacitación y asistencia técnica.

Es de orden constitucional generar el desarrollo rural, segmento propicio para formular emprendimientos en el área agroalimentaria, de ese modo, se encuentra el marco legal fundamental para la generación de leyes, decretos, normas, entre otros instrumentos jurídicos, con el fin de encausar acciones enfocadas al establecimiento de oportunidades para el progreso integral del país desde lo económico.

El orden constitucional vigente, generó la elaboración y puesta en funcionamiento del Plan Nacional de Desarrollo Económico y Social de la Nación (PNDESN) (2001-2007), 


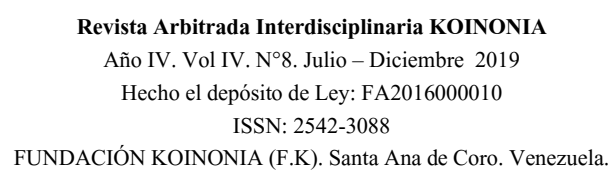

Mariannys Mariely Salón Pereira; Josía Isea

el cual fungía como un plan estratégico a ser desarrollado en el país en aras de generar un "nuevo modelo productivo socialista", el cual basa su enfoque en:

La producción en la búsqueda de lograr trabajo con significado se orientará hacia la eliminación de la división social del trabajo, de su estructura jerárquica actual y a la eliminación de la disyuntiva entre satisfacción de necesidad y producción de riqueza. El modelo productivo responderá primordialmente a las necesidades humanas y estará menos subordinada a la reproducción del capital. (PNDESN, 2001-2007, p. 24).

Un nuevo enfoque económico planteado al país, diverso al modelo tradicional estructurado de la economía capitalista y división de clases, desde esa concepción se posibilitan nuevos estilos de emprendimiento enfocado desde la producción de empresas social, ante lo cual, (PNDESN, 2001-2007), plantea que "El Modelo Productivo Socialista estará conformado básicamente por las Empresas de Producción Social, que constituyen el germen y el camino hacia el Socialismo del Siglo XXI, aunque persistirán empresas del Estado y empresas capitalistas privadas" (p. 24).

Una política emergente propuesta desde lo planteado, en un país donde las empresas primordialmente se estructuraban desde una perspectiva privada capitalista, por consiguiente implica un giro no solo en el paradigma económico, sino, político para germinar así, acciones en pro de articular emprendimientos desde nuevas perspectivas políticas.

Luego se genera el Primer Plan de la Patria Socialista, Proyecto Nacional Simón Bolívar. Desarrollo Económico y Social de la Nación 2007 - 2013. En donde textualmente se mantiene el modelo de producción socialista citado en el plan 2001 2007, lo cual implicaba la profundización de la economía desde el enfoque socialista de economía en preponderancia de la eliminación de la división de clases, para esto es necesario a los emprendedores bajo concepciones ideológicas diversas a las tradicionalmente abordadas en la economía no socialista.

Actualmente se encuentra vigente el Plan de la Patria. Segundo Plan Socialista de Desarrollo Económico y Social de la Nación (2013-2019), en donde se proyectan 


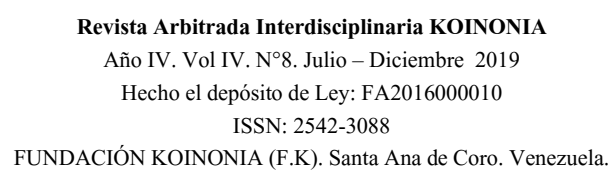

Mariannys Mariely Salón Pereira; Josía Isea

acciones que procuran el avance del sector agroalimentario, entre los cuales se pueden señalar:

1.4.2.7. Incrementar la producción nacional de bioinsumos para impulsar modelos de producción sustentables. 1.4.3. Afianzar un conjunto de políticas públicas de apoyo a la producción, distribución, comercialización y organización del sector rural y participación del poder popular campesino en la implementación de un Plan Nacional de Producción de Alimentos que garantice la soberanía alimentaria. 1.4.3.1. Aumento de la producción nacional agropecuaria (vegetal, pecuaria y acuícola-pesquera) en un $80 \%$, para alcanzar $42 \mathrm{MM}$ de tn/año. 1.4.3.3. Impulsar unas producciones agrícolas sin agro tóxicas, basadas en la diversidad autóctona y en una relación armónica con la naturaleza (p. 9).

La tendencia planteada es hacia la promoción agro ecológica de la producción agroalimentaria, en este sentido, los emprendedores deben ejercer una mirada hacia este segmento a la hora de establecer su plan de negocios, por cuanto esto les permitirá estar en el orden del día en referencia a lo planteado desde las políticas públicas vigentes, de ese modo, podrían existir empresas que favorezcan la conciencia ecológica del mundo.

Prosiguiendo con lo planteado, (Acevedo, 2017, p.1) plantea que "En la Comuna "La Unión" del municipio Pedraza una de sus voceras destacó que sembrarán 2.500 hectáreas de arroz y agradece al Gobierno Bolivariano por el apoyo recibido". El modelo de empresas de producción social (EPS), proyectado en los diversos planes de desarrollo económico de la nación, genera prácticas en el seno de las comunas, organizaciones a las cuales el gobierno nacional ha brindado la oportunidad de otorgar apoyo para la siembra de rubros propios de la zona, sin embargo, no se evidencia si esto ha sido por medio de emprendimiento de las comunidades o por delegación del gobierno para que estas desarrollen una actividad económica agroalimentaria. Por otro lado, (García, 2018) plantea que

Con 53 mil 500 hectáreas productivas y la puesta en marcha de 7.500 conucos integrales inicia este miércoles el Plan de Siembra Comunal. El ministro del Poder Popular para las Comunas y Movimientos Sociales, Aristóbulo Istúriz, detalló que se prevé, en un primer ciclo, arrancar con al 


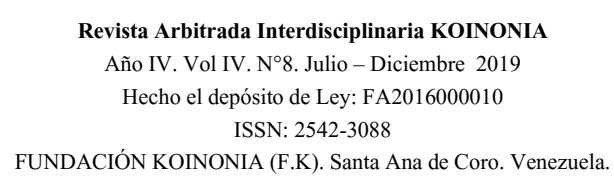

Mariannys Mariely Salón Pereira; Josía Isea

menos 10 hectáreas en el estado portuguesa, destinadas a la producción de cereales. Desde la comuna Chiriguare en el municipio Ospino del estado Portuguesa, el ministro acotó que el Plan de Siembra Comunal se ejecuta en 55 municipios del país, 96 parroquias y 335 comunas. "Hoy arrancamos uno de los pilares fundamental de la economía". Aclaró que en el caso de las comunas que no poseen grandes cantidades de tierra se implementarán los conucos integrales que constan de 2,5 hectáreas y se dedicarán a la producción animal y vegetal. (p. 1).

El modelo productivo socialista desde la política pública es promovido como la alternativa para generar una economía socialista, esto implica la posibilidad de estudiar la germinación de un nuevo enfoque de emprendimiento como podría ser el comunal, es propicio profundizar sobre el tema en futuras investigaciones, desde la experiencia de los actores involucrados con la finalidad de conocer la efectividad del modelo planteado, dado que aún es un proceso en experimentación del cual no se tienen mayores registros investigativos que permitan realizar una confrontación científica, sin embargo, (Romero, 2016) plantea que

El sector agroalimentario venezolano ha transitado con el devenir de los años por acontecimientos históricos que hablan de la realidad actual que le aqueja desde el siglo XX y principios del siglo XXI. Diversas han sido las políticas y estrategias emprendidas, pero las mismas no han logrado superar los problemas existentes, ni mucho menos lograr la seguridad y soberanía alimentaria de los venezolanos, pues se evidencian políticas sin éxito sostenido.

Lo cual se torna en un reto por cuanto es pertinente revisar cómo se han empleado las políticas existentes, si estas han sido generadas desde la aptitud emprendedora, si han recibido la debida asistencia técnica - financiera para desarrollar la idea, son múltiples interrogantes que son pertinentes desarrollarlas a la luz de brindar repuestas científicas al tema, por tanto, hasta ahora se plantea un primer acercamiento desde lo documental para articular ideas que contribuyan al proseguimiento de políticas que favorezcan el emprendimiento agroalimentario y transcender los problemas actuales. 


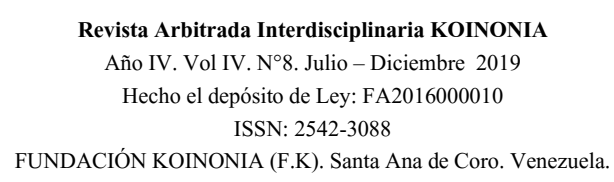

Mariannys Mariely Salón Pereira; Josía Isea

\section{METODOLOGÍA}

El presente artículo fue realizado bajo el enfoque cualitativo, tomando en cuenta los aspectos fundamentales del paradigma interpretativo y la metodología empleada en el actual papel de trabajo es de tipo documental, desarrollada desde el enfoque de Alfonso (1994), quien señala que

La investigación documental es un procedimiento científico, un proceso sistemático de indagación, recolección, organización, análisis e interpretación de información o datos en torno a un determinado tema. Al igual que otros tipos de investigación, éste es conducente a la construcción de conocimientos.

En este sentido, se realizó una revisión de documentos de preponderancia electrónica, lo que permitió generar un acercamiento vigente con el tema planteado, de ese modo, se generó posteriormente un análisis el cual sirve de producto del actual papel de trabajo, es decir, como construcción de conocimientos. Como toda investigación el procedimiento para recopilar y analizar la información partió principalmente de indagar vía recurso electrónico sobre el tema en estudio partiendo de referentes teóricos sobre el emprendimiento, emprendimiento agrícola, rol del emprendedor agrícola, y las políticas públicas que se han generado y sustenta el emprendimiento agrícola en Venezuela. Dichos documentos recopilados fueron organizados y clasificados desde los conceptos básicos hacia la profundidad y complejidad de los temas para su posterior análisis interno del contenido (lectura compresiva de los textos) y así extraer conclusiones.

\section{CONCLUSIONES}

El emprendimiento agroalimentario puede ser generado desde diversos enfoques, estos se pueden desarrollar según la idea de negocio, mercado, rubro, entre otros elementos que debe tener presente el emprendedor a la hora de realizar su plan de inversión, así como adaptarlo a las leyes vigentes que promueven la agroecología como alternativa sustentable para el sostenimiento del ambiente, esto genera potencial de negocio 


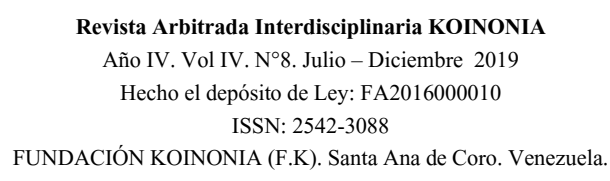

Mariannys Mariely Salón Pereira; Josía Isea

acorde a la sociedad globalizada que se mueve hacia el mercadeo verde y estilos ecológicos de producción.

La soberanía alimentaria se vería beneficiada con el crecimiento de ideas de negocio a partir de emprendimientos que puedan ser apoyados técnica, financieramente, pero sobre todo de acompañamiento formativo hasta que la empresa se encuentre consolidada, de ese modo, se podrá mermar el riesgo de fracaso que tiende a ocurrir sí el emprendedor ejerce acciones al mero azar.

Las políticas públicas actuales se circunscriben al fomento de la comuna como espacio productivo, es necesario realizar investigaciones en este segmento con el fin de conocer científicamente el desarrollo, alcance, beneficios, debilidades, para así, potenciar o tomar correctivos que permitan desde la comuna, afianzar el emprendimiento como un estilo de vida que contribuya a transcender el modelo económico del rentismo petrolero en una economía productiva desde lo endógeno.

Es pertinente incluir en las políticas públicas planes de formación e inversión hacia los emprendedores noveles con la finalidad de promover esta potencialidad como alternativa para generar empresas productivas en las comunidades agrícolas, generando así articulación con las comunas e instituciones públicas y privadas a fin de gestar un emprendimiento efectivo para la economía nacional.

Se deben fomentar en las políticas públicas las herramientas necesarias que permeen la consolidación de los diversos emprendimientos realizados con el fin de proporcionar alimentos y oportunidades de crecimiento económico. Aunado es de vital que las organizaciones encargadas de brindar apoyo a los productores agrícolas estén comprometidos y el personal este capacitados para desarrollar proyectos encaminados a lograr la afianzamiento de la política públicas y por ende el progreso del país.

Por consiguiente, se visualiza el enfoque del emprendimiento de ecosistema como es más viable de ser implementado en la realidad actual venezolana, por cuanto este permite confluir lo público y privado para trabajar sistemáticamente en un plan previamente establecido para el fortalecimiento del sector agroalimentario venezolano. 


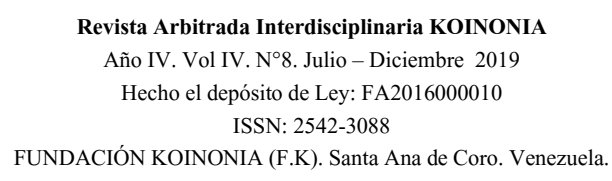

Mariannys Mariely Salón Pereira; Josía Isea

El rol del emprendimiento agrícola es una herramienta fundamental para el progreso del país, por cuanto el emprendedor agrícola debe estar cada día más comprometido en la relación ambiente y el buen aprovechamiento de los recursos naturales para así lograr la productividad y producción sustentable y sostenible. Además el emprendimiento agrícola no solo debe limitarse a la producción constante de un producto; sino que debe ir en busca de nuevas alternativas que permitan mejorar genéticamente la producción y la calidad.

Es necesario que en Venezuela se creen organizaciones que contribuyan a la generación de nuevos mercados y productos agrícolas, donde los productores rurales desarrollen sus capacidades emprendedoras basadas en los conocimientos ancestrales y con la utilización industrial y así las comunidades rurales puedan progresar asiduamente.

\section{REFERENCIAS CONSULTADAS}

1. Acevedo, S. (2017). Plan Integral de Desarrollo Agrícola 2017-2018 fortalece a la Venezuela potencia. Recuperado el día 19/03/2017 de http://fondas.gob.ve/index.ph/plan-integral-de-desarrollo-agricola-2017-2018fortalece-a-la-venezuela-potencia/.

2. Alfonzo, I. (1994). Técnicas de investigación bibliográfica. Caracas: Contexto Ediciones.

3. Brown, T. (2017). El pensamiento de diseño. Recuperado el 15/08/2017 de https://designthinking.ideo.com/?page_id=1542.

4. CONAMYPE (2014). Política nacional de emprendimiento. Recuperado el 08/08/2014 de http://www.conamype.gob.sv/wpcontent/uploads/Poli\%CC\%81 tica-de-Emprendimiento.pdf.

5. Constitución de la República Bolivariana de Venezuela (1999). Capitulo VII. De los Derechos Económicos. Recuperado 23/05/2000 de http://www.asambleanacional.gob.ve/documentos archivos/constitucionnacional-7.pdf.

6. Constitución de la República Bolivariana de Venezuela (1999). Capitulo IX. De los Derechos

Ambientales.

Recuperado 


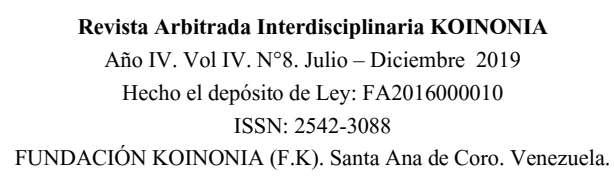

Mariannys Mariely Salón Pereira; Josía Isea

http://www.asambleanacional.gob.ve/documentos archivos/constitucionnacional-7.pdf.

7. Constitución de la República Bolivariana de Venezuela (1999). Del régimen socioeconómico y de la función del Estado en la economía. Recuperado de http://www.asambleanacional.gob.ve/documentos archivos/constitucionnacional-7.pdf.

8. García, M. (2018). Plan de Siembra Comunal inicia con 53 mil 500 hectáreas productivas. Recuperado de http://www.correodelorinoco.gob.ve/plan-desiembra-comunal-inicia-con-53-mil-500-hectareas-productivas/.

9. Giacalone, R., Hernández, M., y Zerpa, S. (2010). El financiamiento del componente primario del sistema agroalimentario venezolano. La visión de los actores privados. Revista Agroalimentaria. Vol. 16, № 30. Enero-junio 2010 (167189).

Recuperado http://erevistas.saber.ula.ve/index.php/agroalimentaria/article/view/1483.

10. Heynig, K. (1982). Principales Enfoques Sobre La Economía Campesina. Revista de la CEPAL no 16, 1982. Recuperado de https://repositorio.cepal.org/bitstream/handle/11362/10332/016115142 es.pdf?se quence $=1$ \&is Allowed $=\mathrm{y}$.

11. Marulanda, J. Correa, G., y Mejía, L. (2009). Emprendimiento: Visiones desde las teorías del comportamiento humano. Revista Escuela de Administración de Negocios, núm. 66, mayo-agosto, 2009 pp. 153-168. Recuperado de https://www.redalyc.org/pdf/206/20620269008.pdf.

12. Navarro Prado, M., Aguilar, L., López Sampson, A. Breuer, B. (2013). Fomento de la cadena de valor de cacao en Nicaragua: Cooperación Alemania 2000 2012. Recuperado de https://censalud.ues.edu.sv/CDOCDeployment/documentos/sistematizacionFomento de la cadena de valor de cacao Nicaragua.pdf.

13.Nuñez Torreón, A. (2017). Que son y en qué consisten los ecosistemas emprendedores. Recuperado de http://www.ticbeat.com/empresa-b2b/que-sony-en-que-consisten-los-ecosistemas-emprendedores/

14. Osterwalder, A., y Pigneur, Y. (2017). Generación de modelos de negocio. Recuperado de https://inaem.aragon.es/sites/default/files/TALLER 5 Generacion de Modelos d e Negocios.pdf. 
15. Peña Aldazoro, O. (2018). Política Alimentaria, Inclusión social y cambios en los estratos sociales en Venezuela. Revista Arbitrada Interdisciplinaria Koinonía, 2(4), 60-83. Recuperado de http://fundacionkoinonia.com.ve/ojs/index.php/revistakoinonia/article/view/70.

16. Plan Nacional de Desarrollo Económico y Social de la Nación (PNDESN) (20012007). IV Modelo productivo socialista. Recuperado de http://www.mppp.gob.ve/wp-content/uploads/2013/09/Plan-de-la-Naci\%C3\%B3n2007-2013.pdf.

17.Primer Plan de la Patria Socialista, Proyecto Nacional Simón Bolívar. Desarrollo Económico y Social de la Nación 2007 - 2013. IV Modelo productivo socialista. Recuperado de https://www.mppeuct.gob.ve/sites/default/files/descargables/proyecto-nacionalsimon-bolivar.pdf.

18. Plan de la Patria. Segundo Plan Socialista de Desarrollo Económico y Social de la Nación (2013-2019). Gran objetivo histórico $N^{\circ} 1$. Recuperado de http://www.mppp.gob.ve/wp-content/uploads/2013/09/ley plan patria.pdf.

19.Romero, M. (2016). Políticas y estrategias en el sector agroalimentario venezolano. Implicaciones sociales. Recuperado de http://www.produccioncientifica.luz.edu.ve/index.php/rcs/article/view/22800/2268.

20.Taborda, L., y Sosa, M. (2014). Un modelo de emprendimiento agrícola a partir de educación superior rural en la Colombia profunda. Recuperado de https://dialnet.unirioja.es/descarga/articulo/4865368.pdf 


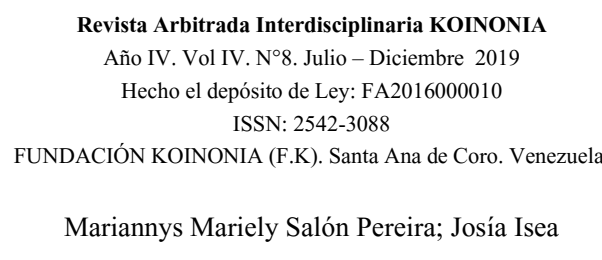

\section{CONSULTED REFERENCE}

1. Acevedo, S. (2017). Comprehensive Plan for Agricultural Development 20172018 strengthens Venezuela's power. Retrieved from http://fondas.gob.ve/index.php/2017/03/19/plan-integral-de-desarrollo-agricola2017-2018-fortalece-a-la-venezuela-potencia/.

2. Alfonzo, I. (1994). Bibliographic research techniques. Caracas: Context Editions.

3. Brown, T. (2017). The design thinking. Retrieved from https://designthinking.ideo.com/?page id=1542.

4. CONAMYPE (2014). National entrepreneurship policy. Retrieved from http://www.conamype.gob.sv/wp-content/uploads/2014/08/Poli\%CC\%81tica-de-

Emprendimiento.pdf.

5. Constitution of the Bolivarian Republic of Venezuela (1999). Chapter VII. Of the Economic Rights. Retrieved from http://www.asambleanacional.gob.ve/documentos archivos/constitucionnacional-7.pdf.

6. Constitution of the Bolivarian Republic of Venezuela (1999). Chapter IX. Of Environmental Rights. Retrieved from http://www.asambleanacional.gob.ve/documentos archivos/constitucionnacional-7.pdf.

7. Constitution of the Bolivarian Republic of Venezuela (1999). Of the socioeconomic regime and of the function of the State in the economy. Retrieved from http://www.asambleanacional.gob.ve/documentos archivos/constitucionnacional-7.pdf.

8. García, M. (2018). Communal Planting Plan starts with 53 thousand 500 productive hectares. Retrieved from http://www.correodelorinoco.gob.ve/plan-desiembra-comunal-inicia-con-53-mil-500-hectareas-productivas/.

9. Giacalone, R., Hernández, M., and Zerpa, S. (2010). The financing of the primary component of the Venezuelan agro-food system. The vision of private actors. Agrifood Magazine. Vol. 16, No. 30. January-June 2010 (167-189). Retrieved from http://erevistas.saber.ula.ve/index.php/agroalimentaria/article/view/1483.

10. Heynig, K. (1982). Main Approaches on the Peasant Economy ECLAC Magazine No.

16 , 1982.

Retrieved from 


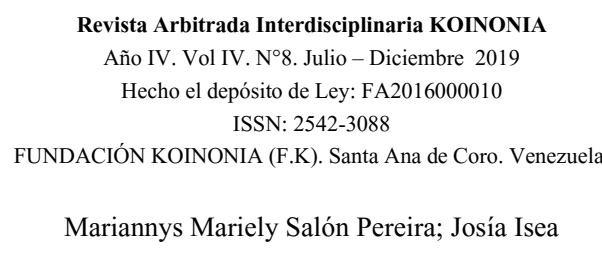

https://repositorio.cepal.org/bitstream/handle/11362/10332/016115142 en.pdf?se quence $=1$ \&isAllowed $=\mathbf{y}$.

11. Marulanda, J. Correa, G., and Mejía, L. (2009). Entrepreneurship: Visions from theories of human behavior. School of Business Administration Magazine, no. 66, May-August, 2009 pp. 153-168. Retrieved from https://www.redalyc.org/pdf/206/20620269008.pdf.

12. Navarro Prado, M., Aguilar, L., Lopez Sampson, A. Breuer, B. (2013). Promotion of the cocoa value chain in Nicaragua: Cooperation Germany 2000 - 2012. Retrieved from https://censalud.ues.edu.sv/CDOCDeployment/documentos/sistematizacionFomento de la cadena de valor de cacao Nicaragua.pdf.

13.Nuñez Torreón, A. (2017). What are the entrepreneurial ecosystems? Recovered from http://www.ticbeat.com/empresa-b2b/que-son-y-en-que-consisten-losecosistemas-emprendedores/

14. Osterwalder, A., and Pigneur, Y. (2017). Generation of business models. Retrieved from https://inaem.aragon.es/sites/default/files/TALLER 5 Generacion de Modelos d e Negocios.pdf

15.Peña Aldazoro, O. (2018). FOOD POLICY, SOCIAL INCLUSION AND CHANGES IN SOCIAL STRATEGIES IN VENEZUELA. Journal Interdisciplinary Arbitrated Koinonía, 2 (4), 60-83. Retrieved from http://fundacionkoinonia.com.ve/ojs/index.php/revistakoinonia/article/view/70.

16. National Plan for Economic and Social Development of the Nation (PNDESN) (2001-2007). IV Socialist productive model. Retrieved from http://www.mppp.gob.ve/wp-content/uploads/2013/09/Plan-de-la-Naci\%C3\%B3n2007-2013.pdf.

17. First Plan of the Socialist Homeland, Simón Bolívar National Project. Economic and Social Development of the Nation 2007 - 2013. IV Socialist productive model. Retrieved from https://www.mppeuct.gob.ve/sites/default/files/descargables/proyecto-nacionalsimon-bolivar.pdf.

18. Plan of the Fatherland. Second Socialist Plan for Economic and Social Development of the Nation (2013-2019). Great historical objective No. 1. 
Revista Arbitrada Interdisciplinaria KOINONIA

Año IV. Vol IV. №8. Julio - Diciembre 2019

Hecho el depósito de Ley: FA2016000010

ISSN: 2542-3088

FUNDACIÓN KOINONIA (F.K). Santa Ana de Coro. Venezuela.

Mariannys Mariely Salón Pereira; Josía Isea

content/uploads/2013/09/ley_plan_patria.pdf.

http://www.mppp.gob.ve/wp-

19. Romero, M. (2016). Policies and strategies in the Venezuelan agro-food sector. Social implications

Retrieved

from

http://www.produccioncientifica.luz.edu.ve/index.php/rcs/article/view/22800/2268.

20.Taborda, L., and Sosa, M. (2014). A model of agricultural entrepreneurship based on rural higher education in deep Colombia. Retrieved from https://dialnet.unirioja.es/descarga/articulo/4865368.pdf.

C2019 por los autores. Este artículo es de acceso abierto y distribuido según los términos y condiciones de la licencia Creative Commons Atribución-NoComercial-Compartirlgual 4.0 Internacional (CC BY-NC-SA 4.0)

(https://creativecommons.org/licenses/by-nc-sa/4.0/). 